\title{
Individual-Level and Population-Level Lateralization: Two Sides of the Same Coin
}

\author{
Elisa Frasnelli ${ }^{1, *}$ and Giorgio Vallortigara ${ }^{2}$ (D) \\ 1 School of Life Sciences, University of Lincoln, Lincoln LN6 7DL, UK \\ 2 Center for Mind/Brain Sciences, University of Trento, Piazza della Manifattura 1, I-38068 Rovereto, Italy; \\ giorgio.vallortigara@unitn.it \\ * Correspondence: efrasnelli@lincoln.ac.uk
}

Received: 21 November 2018; Accepted: 7 December 2018; Published: 11 December 2018

check for updates

\begin{abstract}
Lateralization, i.e., the different functional roles played by the left and right sides of the brain, is expressed in two main ways: (1) in single individuals, regardless of a common direction (bias) in the population (aka individual-level lateralization); or (2) in single individuals and in the same direction in most of them, so that the population is biased (aka population-level lateralization). Indeed, lateralization often occurs at the population-level, with $60-90 \%$ of individuals showing the same direction (right or left) of bias, depending on species and tasks. It is usually maintained that lateralization can increase the brain's efficiency. However, this may explain individual-level lateralization, but not population-level lateralization, for individual brain efficiency is unrelated to the direction of the asymmetry in other individuals. From a theoretical point of view, a possible explanation for population-level lateralization is that it may reflect an evolutionarily stable strategy (ESS) that can develop when individually asymmetrical organisms are under specific selective pressures to coordinate their behavior with that of other asymmetrical organisms. This prediction has been sometimes misunderstood as it is equated with the idea that population-level lateralization should only be present in social species. However, population-level asymmetries have been observed in aggressive and mating displays in so-called "solitary" insects, suggesting that engagement in specific inter-individual interactions rather than "sociality" per se may promote population-level lateralization. Here, we clarify that the nature of inter-individuals interaction can generate evolutionarily stable strategies of lateralization at the individual- or population-level, depending on ecological contexts, showing that individual-level and population-level lateralization should be considered as two aspects of the same continuum.
\end{abstract}

Keywords: lateralization; individual-level; population-level; evolution; ESS; social interactions

\section{Introduction}

Lateralization, defined as the different specialization of function of the left and right sides of the nervous system, is a widespread phenomenon in the animal kingdom. In the last three decades, many studies have provided evidence that many animal species, from the evolutionarily closest to the most evolutionarily distant from humans, show asymmetrical biases in behavior [1]. Examples range from the asymmetrical use of limbs to handle objects or perform motor activities (for a review, see [2]) to the asymmetrical use of sensory pair organs, such as eyes, nostrils, ears, and antennae to detect a specific stimulus, such as a potential predator; from motor biases in escape directions or navigation to the asymmetrical processes involving learning and memory and the process of emotions $[3,4]$. All this evidence of brain and behavioral asymmetries in vertebrates [1], together with some in invertebrates $[5,6]$, suggests that having an asymmetrical brain must confer advantages to complex brains, as well as to "simpler" ones. 
Lateralization varies in strength (an individual may be less or more strongly lateralized) and direction (left or right) among individuals of the same species, of different species, and also depending on the task considered. Moreover, it can be present at the individual- or population-level (when most individuals within the population show the same direction of bias). Population-level lateralization has been explained as a consequence of selective social pressures that have pushed individuals to coordinate with each other and align their biases in the same direction [7]. In this paper, we discuss the advantages and disadvantages connected with having a (less or more) strong lateralized brain and the complexity of this fascinating phenomenon, while claiming that individual-level and population-level lateralization should be interpreted as two aspects of the same continuum.

\section{Advantages of Having an Asymmetrical Brain (at the Individual Level)}

Having an asymmetrical brain provides several advantages (see, for an extensive discussion, [7-9]). If the left and right sides of the brain perform different functions, it is possible to save energetic resources in cognitive tasks. Indeed, lateralization allows avoiding the duplication of functions in the two hemispheres (otherwise, animals should probably have a brain double the size). Another big advantage related to lateralization consists of the possibility to separately and simultaneously process external stimuli, increasing the efficiency of the cerebral capacity. This is particularly easy to observe in animals with laterally placed eyes, such as birds, which mainly have monocular vision when using their lateral visual fields, i.e., they use their right and their left eye separately. More precisely, in birds, the lateral part of the right retina only communicates with the left hemisphere and vice versa. Because of this peculiarity, species such as the domestic chick Gallus gallus have been widely studied to assess the preferential use of the left and right side of the brain in specific tasks $[10,11]$. Chicks are better at discriminating grains of food from pebbles randomly mixed on the ground when they use their right eye (and thus their left hemisphere, as in vertebrates, the left hemisphere controls the right part of the body and vice versa; [12] see also for quails [13]). At the same time, chicks are better at detecting the presence of a potential predator when this appears in their left visual hemi-field (and it is perceived by their left eye and thus by the right hemisphere; [12]). Because of this functional specialization, chicks can feed from the ground using their right eye and, simultaneously, they can keep their left eye ready to respond to and protect themselves from potential predators [14].

Furthermore, when one hemisphere controls a specific behavior (for example, detecting potential predators), it is not competing with the other hemisphere to take control of that specific behavior. This leads to a more rapid and efficient response. Cerebral lateralization is indeed linked to better cognitive performances. Some studies have shown that more strongly lateralized individuals are more successful in some cognitive tasks compared to weakly lateralized conspecifics. In fact, behavioral asymmetries may vary not only in direction, but also in strength, among different individuals of the same species: some individuals can be more or less left-biased, others right-biased, and yet others unbiased. This is the case, for example, for chimpanzees, when fishing termites using a stick: individuals with a strong preference to consistently use one hand (regardless of whether it is the left or the right one) are more efficient than individuals that do not have any preference to use one or the other hand [15]. Children with consistent early hand preferences exhibit advanced patterns of cognitive development compared to children who develop a hand preference later, although this could be a matter of synchronized development [16]. Strongly lateralized parrots showing a significant foot and eye preference are better at solving novel problems, such as a pebble-seed discrimination test and a string-pull problem, than less strongly lateralized parrots [17]. In domestic chicks (Gallus gallus), a right-eye superiority has been documented in inhibiting pecks at pebbles while searching for grain and this ability is impaired when lateralization is not present [14,18]. Similarly, pigeons (Columba livia domestica) with the strongest eye lateralization in discriminating grains from pebbles are the most successful in selecting grains when tested binocularly, suggesting that stronger lateralization increases the efficiency of a performance [19]. 
Surprisingly, insects also seem to have a preference for using one limb. Locusts crossing a gap have been shown to preferentially use the left or the right leg in this task [20]. Different individuals showed different biases not only in the direction (left or right), but also in the strength of the bias. However, as in chimpanzees [15], the individuals with a strong preference were those that made fewer mistakes in the task and thus were most successful [21]. This suggests that in this specific context, stronger lateralization confers a benefit in terms of improved motor control. Strong lateralization also seems to influence learning ability, as shown in larval antlions (Myrmeleon bore), with strong lateralized righting behavior being better at associating a vibrational cue with prey removal [22].

Not only behavioral asymmetries may vary in direction and strength among different individuals of the same species; biases can also change, depending on the task that an animal is performing (e.g., handedness in marmosets, [23]). This indicates that lateralization is a complex phenomenon that varies at the species, group, and individual level, bringing us to the question of what are the advantages of having individuals with different biases in the population. Individuals with a strong lateralization seem to have an advantage in terms of improved motor control $[15,21]$ or problem solving [14,17-19]. However, in strongly lateralized fish, a consistent lateral bias to turn in one direction reduces their ability to orient in a maze [24]. This makes the scenario more complex and opens further questions about the optimal degree (and direction) of bias that an individual should have, depending on the task and functional context.

In sage grouse (Centrocercus urophasianus), successfully mating males are in general more strongly lateralized in courtship behavior than non-mating males, but this depends on the behavior of the male and the social environment in which he is acting [25]. Larger male fallow deer (Dama dama) display a greater tendency to show a right-sided bias when terminating the parallel walk during fights and terminated parallel walks sooner than smaller individuals, suggesting that lateralization provides a mechanism by which contestants can resolve contests at a low cost [26]. Accordingly, in dyadic contests, domestic pigs (Sus scrofa) with strong lateralization in the orientation towards their opponent (regardless of the direction) have a shorter contest duration than conspecific, with a weak bias. However, although lateralization seems to play a role in conflict resolution, it does not influence fighting success, as winners and losers showed a similar strength and direction of bias [27]. Less lateralized wild elk (Cervus canadensis) for front-limb biases (i.e., handedness) respond more intensely to aversive stimuli (predator-resembling chases by humans), but the same animals are also more inclined to reduce their flight responses (i.e., habituate) to human approaches when benign [28]. On the other hand, more lateralized elks are bolder and more likely to move around, whereas less lateralized animals tend to remain near humans year-round [28].

Substantial individual variation in the strength of cerebral lateralization may be associated with individual variation in behaviour. For example, non-lateralized domestic chicks emitted more distress calls and took longer to resume pecking at food after exposure to a simulated predator than lateralized chicks [29]. Strongly lateralized convict cichlids (Amatitlania nigrofasciata) are quicker to emerge from a refuge indicative of boldness [30]. The degree of laterality seems to be positively correlated with stress reactivity in Port Jackson sharks (Heterodontus portusjacksoni) [31].

Recently, Whiteside and colleagues [32] showed that pheasants with a strong foot preference in motor tasks were more likely to die earlier in natural conditions than conspecific, with a mild foot preference. This study is the first trying to link lateralization with fitness in terms of survival and seems to suggest that the degree of lateralization does not linearly associate with benefits and that there is an optimum degree of laterality for pheasants in order to get the highest fitness (i.e., survival). Indeed, as stated by Rogers, Vallortigara and Andrew [1], arguing for computational advantages associated with the possess of an asymmetrical brain is not the same as arguing that the more asymmetric a brain, the more computationally-efficient it will be. In humans, there is a clear inverted U-shape curve in the relationship between degree of laterality and performance in word matching and face decision tasks [33], suggesting that a moderately asymmetrical brain would provide the greatest advantage. Finally, the relationship between lateralization and performance is task dependent [34]; therefore, a 
degree of laterality that may benefit one task may not benefit another. Survival requires an individual to detect predators, discriminate and handle food, cope with disease, navigate a complex environment, and learn strategies and much work on proxy measures of fitness looks at single factors, often in highly controlled environments.

\section{Population-Level Lateralization as an Evolutionarily Stable Strategy (ESS)}

Lateralization presents an intriguing aspect: it is often present at the population-level (i.e., directional asymmetry, where more than $50 \%$ of individuals within a population show the same direction of bias, such as handedness in humans, where about $90 \%$ of people are right-handed; [35]). If lateralization confers several advantages to the single individual in terms of brain efficiency, this cannot explain the alignment of the bias in the population.

The first evidence for a role of social behavior in population-level lateralization was provided by Rogers and Workman [36], who showed that more strongly lateralized chicks acquire a higher position in the social hierarchy than less lateralized chicks. Subsequently, Vallortigara and Rogers [7] reviewed the overall evidence and argued for a role of social interaction in the evolution of population-level brain asymmetry. The hypothesis was supported by a theoretical model developed by Ghirlanda and Vallortigara ([37]; see also [38]) showing that, in the context of prey-predator interactions, population-level lateralization can develop as an evolutionarily stable strategy (ESS) when individually asymmetrical organisms must coordinate their right-left behavioral patterns with those of other asymmetrical organisms. As a lateralized brain leads to behavioral biases when escaping from predators (e.g., [39]), the model considered the fitness consequences that the lateralization of one prey has when it interacts with other group-living prey subject to predation. The model assumed that the fitness was influenced by two contrasting selection pressures: (1) the benefit of being lateralized in the direction of the majority as a consequence of the "dilution effect" (i.e., prey in large groups have a lesser risk of being targeted by predators; [40]); and (2) the cost of being lateralized in the direction of the majority as a consequence of predators learning to anticipate prey escape strategies. In this second case, individuals who escape in a different direction from the majority have a benefit as they can surprise predators and survive more often. By varying the contribution of these costs and benefits, the model showed that population-level lateralization emerges as an ESS when neither of the two selection pressures is much stronger than the other. Thus, the successful strategy of group-living prey is to have a majority of individuals gaining protection from the group and escaping in the same direction when facing a predator and a minority of them being able to surprise the predator by escaping in the opposite direction. Empirical support for this hypothesis comes from fish schools, where animals showing the same turning bias as the majority of the group have an improved escape performance than fish at odds with the group [41].

A few years later, the mathematical model by Ghirlanda and Vallortigara [37] was extended by considering intraspecific interactions instead of interspecific prey-predator interactions [42]. Specifically, the new model considered the selective pressures of synergistic (cooperative) and antagonistic (competitive) interactions on individuals being lateralized in the same or opposite direction within the same species. It assumed that individuals lateralized in the same direction have a benefit in engaging in synergistic interactions as they can, for example, efficiently use the same tools or coordinate better. On the other side, individuals lateralized in the direction different from that of the majority have an advantage when engaging in antagonistic interactions for the same reason as in the previous model: they can surprise the opponent by adopting a strategy to which opponents are less accustomed. Empirical support for this assumption comes from the success of left-handers (i.e., lateralized in the opposite direction compared to the majority) in competitive sports such as fencing, boxing, and tennis (e.g., [43]; see also [44]). The ESS model for intraspecific interactions [42] showed that when the pressure of synergistic interactions becomes more and more important compared to that of antagonistic interactions, individually asymmetric organisms must interact with conspecifics 
and coordinate their activities and, consequently, asymmetry aligns in the majority of individuals in a population (i.e., directional or population-level asymmetry).

In order to provide empirical evidence for this prediction, the relationship between the level of lateralization and the presence of social behaviors was investigated using different species of bees as a model system (summarized in [45]; see also [46]). A series of experiments provided striking evidence that the alignment of lateralization within the population may be a consequence of social interactions frequently encountered during the course of evolution [47-49]. In fact, eusocial honeybees Apis mellifera [47], three species of primitively social Australian stingless bees [48], and annual social bumblebees Bombus terrestris [49], but not the solitary bees Osmia rufa [47], were found to be asymmetrical at the population-level for the use of the left and right antennae in recalling olfactory memories. However, all these studies investigated the use of a preferred antenna in recalling a learnt memory of an association between an odor and a food reward, and not really social interactions.

The first evidence of the role of antennal asymmetries in social interactions was shown in highly social ants Formica rufa [50]. By looking at "feeding" contacts where a "donor" ant exchanges food with a "receiver" ant through trophallaxis, the researchers [50] observed population-level asymmetry, with the "receiver" ant using the right antenna more frequently than the left antenna. The role of antennal asymmetries has also been investigated by observing the behavior of different dyads of honeybees with only the left, only the right, or both antennae in use, and belonging to the same or different hives [51]. In bees belonging to the same hive, dyads having only the right antenna in use took less time to get in contact and interacted more positively then dyads with only the left antennae, which instead interacted more aggressively than the other two groups. Interestingly, for bees belonging to different hives, dyads with only the right antenna in use displayed more aggressive interactions than bees with only the left or both antennae [51]. This suggests that the right antenna seems to control the correct behavioral response, depending on the social context, i.e., positive interactions between individuals of the same colony and negative interactions between individuals belonging to different colonies. A similar pattern of behavior between individuals of the same colony has been found in primitively social stingless bees Trigona carbonaria, where the right antenna stimulates positive contact and the left stimulates avoidance or attack [52].

Advantages of the population-level lateral bias have also been documented in the preference for keeping the mother on the left side in several terrestrial and aquatic mammal infants, supporting the idea of the role that lateralization plays in social interaction [53].

\section{Individual- or Population-Level Lateralization as an ESS}

Only recently, however, our research provided surprising findings: not only social species, but also so-called "non-social" species, of insects show asymmetries at the population-level when their limited interactions with others individuals are considered. This is the case for Osmia rufa, a species that does not show behavioral asymmetry in the recall of short-term olfactory memory [47], but shows population-level lateralization in aggressive displays [54], similarly to eusocial honeybees [51] and social stingless bees T. carbonaria [52]. Clearly, being engaged in interactions with other individuals, rather than the way in which the species nests (socially or not), may affect lateralization.

In honeybees, so far, all the identified biases occur at the population-level: in the use of the right visual pathway to learn visual stimuli [55], in the different use of the antennae in learning and recall of olfactory memories [56-58], and in context-dependent social interactions with conspecifics [51]. A recent study, however, suggests that honeybees tested in a tunnel with gaps of different apertures to the right and left sides, do not show population-level lateralization [59]. In this task, some individuals showed a bias to the right, some others a bias to the left, and yet others no bias. This may indicate that behavioral biases in bees vary in strength and direction, depending on whether the task requires coordination among individuals. Note, however, that very few bees showed individual bias in this task, and thus it is not clear whether individual lateralization was observed. Another example may be provided by foragers of $F$. pratensis ants, a species which does not use trail pheromones, moves more 
often to the left side than to the right whilst walking towards the nest, and does not show any bias when leaving the nest [60]. Moreover, it is still not clear to what extent the alignment occurs. Red wood ants Formica rufa belonging to different colonies show population-level biases in different directions when tested for forelimb preference during a gap crossing task, suggesting that social pressures act to coordinate individuals within the same colony and not necessarily at the species-level [61].

The different types of social interaction can generate evolutionarily stable strategies of lateralization at the individual- or population-level, depending on ecological contexts. Indeed, as we showed, population-level asymmetries have been observed in aggressive and mating displays in so-called "solitary" insects (e.g., tephrid flies, [62]; mason bees, [54]), suggesting that engagement in specific inter-individual interactions rather than "sociality" in general may generate population-level lateralization. This implies that lateralization is not necessarily a static feature of the neural organization, but is modulated by the functional context. For example, the nematode $C$. elegans exhibits a pronounced motor bias: males show a right-turning population-bias during mating. Interestingly, this motor bias is also observed in nematodes with mirror-reversed anatomical asymmetry, perhaps driven by epigenetic factors rather than by genetic variation [63].

The hypothesis that lateralization arises as an ESS is general and thus can predict either population- or individual-level lateralization, depending on the type of interactive behavior considered (e.g., cooperative or competitive) and ecological context. Although the advantage of being aligned in the same direction is clear in cooperative behavior, it is not in aggressive interactions. Indeed, it may be more advantageous for an aggressive display to not be directional, since population-level bias would also mean predictability [7]. For example, if an individual attacks another individual, it would be more convenient for it to be unpredictable. As a consequence, although each individual would have an (individual-level) bias, there will be 50:50 right:left-biased individuals in the population. This is the case for some predators, such as sailfish, which are lateralized at the individual-level in attacking schooling sardines on one side (and the stronger they are lateralized, the more successful they are at capturing their prey), but that overall, do not show a population-level bias [64]. However, if we think specifically about aggressive displays (and not the interactions), the alignment within the population may be linked to the need of an individual to position itself in a congruent way from a postural/motor point of view, as happens in mating (for a review, see [65]).

If being aligned in the same direction may help individuals to better coordinate with each other in specific tasks that require coordination between two or more individuals, being more or less biased in opposite directions may also have a potential benefit in other tasks where it is important to make best use of the available resources. This is something that future studies should address.

\section{Conclusions}

The ESS remains the single most powerful and widespread evolutionary hypothesis to explain lateralization. The ESS theoretical models [37,42] are well-supported by the new data showing population-level lateralization in interactions in the so-called "solitary" insects [54] and individual-level lateralization in social insects for tasks not requiring coordination [59], as the models predict that when social pressures become higher, population-level lateralization arises.

It is important, however, in order to avoid misunderstanding of the theory, to distinguish the claims of the ESS theory as an evolutionary hypothesis (i.e., in terms of natural history) and the claims concerning current living organisms. In terms of natural history, the ESS model hypothesizes that individual-level lateralization emerged first (because of the computational advantages associated with the individual possession of a slight asymmetry between the two halves of the brain) and that an alignment in the direction of the asymmetries evolved subsequently as a result of the interactions between individually-asymmetric organisms. For current-living organisms, any neat distinction between social and non-social species is obviously meaningless because definitions attain to the formal convention of specific disciplines. In entomology, honeybees are social and mason bees are solitary. But of course, this does not mean that mason bees do not interact with conspecifics. Thus, for the 
EES theory, the crucial issue is not the abstract definition of a species as social or not social, but rather whether a specific lateralized behavior entails constraints associated with the presence of other individuals performing the same lateralized behavior.

Importantly, the theory does not predict in a simplistic way that all living species that are not "social" should be lateralized at the individual level and that those that are "social" should be lateralized at the population level. What is important is the presence of inter-individual interactions in which the asymmetry of the individuals influences that of others (e.g., aggressive interactions in mason bees, [54]). In other words, a major prediction of ESS theory is that alignment in lateralization should be expected whenever asymmetric individuals exhibit a benefit from coordination with others asymmetric individuals which is higher than the cost associated with the predictability of their individual behavior. Vice versa, the lack of alignment in lateralization should be expected whenever costs associated with the predictability of individual behavior overcome the benefit of coordinating the behavior among different asymmetric individuals. This is a testable hypothesis that holds true, irrespective of whether individuals of a species are conventionally defined as "social" or "solitary".

For the ESS theory, individual-level and population-level lateralization are the two sides of the same coin or, even better, of the same continuum: the stability (i.e., an ESS) can be obtained with an individual-level or population-level asymmetry, depending on the context. In other words, the theory does not predict that social species need to be lateralized at the population-level, but rather that individual-level or population-level lateralization emerges as an ESS. Indeed, as discussed above, there may be cases in which, within the group, it is stable to be asymmetrical at the individual level (e.g., [64]).

Author Contributions: E.F. and G.V. conceived the paper, E.F. wrote the paper with inputs and additions from G.V. Funding: This research received no external funding.

Acknowledgments: We thank Lesley Rogers for inviting us to contribute this paper in this special issue of Symmetry.

Conflicts of Interest: The authors declare no conflicts of interest.

\section{References}

1. Rogers, L.J.; Vallortigara, G.; Andrew, R. Divided Brains: The Biology and Behaviour of Brain Asymmetries, 1st ed.; Cambridge UP: Cambridge, UK, 2013.

2. Versace, E.; Vallortigara, G. Forelimb preferences in human beings and other species: Multiple models for testing hypotheses on lateralization. Front. Psychol. 2015, 6, 233. [CrossRef]

3. Rogers, L.J.; Vallortigara, G. When and Why Did Brains Break Symmetry? Symmetry 2015, 7, 2181-2194. [CrossRef]

4. Vallortigara, G.; Versace, E. Laterality at the Neural, Cognitive, and Behavioral Levels. In APA Handbook of Comparative Psychology: Vol. 1. Basic Concepts, Methods, Neural Substrate, and Behavior; American Psychological Association: Washington, DC, USA, 2017; pp. 557-577.

5. Frasnelli, E.; Vallortigara, G.; Rogers, L.J. Left-right asymmetries of behavioural and nervous system in invertebrates. Neurosci. Biobehav. Rev. 2012, 36, 1273-1291. [CrossRef]

6. Frasnelli, E. Brain and behavioral lateralization in invertebrates. Front. Psychol. 2013, 4, 1-10. [CrossRef]

7. Vallortigara, G.; Rogers, L.J. Survival with an asymmetrical brain: Advantages and disadvantages of cerebral lateralization. Behav. Brain Sci. 2005, 28, 575-588. [CrossRef]

8. Vallortigara, G.; Rogers, L.J.; Bisazza, A. Possible evolutionary origins of cognitive brain lateralization. Brain Res. Rev. 1999, 30, 164-175. [CrossRef]

9. Vallortigara, G. Comparative neuropsychology of the dual brain: A stroll through left and right animals' perceptual worlds. Brain Lang. 2000, 73, 189-219. [CrossRef]

10. Andrew, R.J. (Ed.) Neural and Behavioural Plasticity: The Use of the Domestic Chicken as a Model; Oxford University Press: Oxford, UK, 1991. 
11. Vallortigara, G.; Cozzutti, C.; Tommasi, L.; Rogers, L.J. How birds use their eyes: Opposite left-right specialisation for the lateral and frontal visual hemifield in the domestic chick. Curr. Biol. 2001, 11, $29-33$. [CrossRef]

12. Rogers, L.J.; Anson, J.M. Lateralisation of function in the chicken forebrain. Pharmacol. Biochem. Behav. 1979, 10, 679-686. [CrossRef]

13. Valenti, A.; Sovrano, V.A.; Zucca, P.; Vallortigara, G. Visual lateralization in quails. Laterality 2003, 8, 67-78. [CrossRef]

14. Rogers, L.J.; Zucca, P.; Vallortigara, G. Advantage of having a lateralized brain. Proc. R. Soc. Lond. B 2004, 271, S420-S422. [CrossRef]

15. McGrew, W.C.; Marchant, L.F. Laterality of hand use pays off in foraging success for wild chimpanzees. Primates 1999, 40, 509-513. [CrossRef]

16. Marcinowski, E.C.; Campbell, J.M.; Faldowski, R.A.; Michel, G.F. Do hand preferences predict stacking skill during infancy? Dev. Psychobiol. 2016, 58, 958-967. [CrossRef]

17. Magat, M.; Brown, C. Laterality enhances cognition in Australian parrots. Proc. R. Soc. Lond. B 2009, 276, 4155-4162. [CrossRef]

18. Rogers, L.J. Evolution of hemispheric specialisation: Advantages and disadvantages. Brain Lang. 2000, 73, 236-253. [CrossRef]

19. Güntürkün, O.; Diekamp, B.; Manns, M. Asymmetry pays: Visual lateralization improves discrimination success in pigeons. Curr. Biol. 2000, 10, 1079-1081. [CrossRef]

20. Bell, A.T.; Niven, J.E. Individual-level, context-dependent handedness in the desert locust. Curr. Biol. 2014, 24, R382-R383. [CrossRef]

21. Bell, A.T.; Niven, J.E. Strength of forelimb lateralization predicts motor errors in an insect. Biol. Lett. 2016, 12, 20160547. [CrossRef]

22. Miler, K.; Kuszewska, K.; Woyciechowski, M. Larval antlions with more pronounced behavioural asymmetry show enhanced cognitive skills. Biol. Lett. 2017, 13, 20160786. [CrossRef]

23. Hook, M.A.; Rogers, L.J. Visuospatial reaching preferences of common marmosets (Callithrix jacchus): An assessment of individual biases across a variety of tasks. J. Comp. Psychol. 2008, 122, 41-51. [CrossRef]

24. Brown, C.; Braithwaite, V.A. Effects of predation pressure on the cognitive ability of the poeciliid Brachyraphis episcopi. Behav. Ecol. 2005, 16, 482-487. [CrossRef]

25. Krakauer, A.H.; Blundell, M.A.; Scanlan, T.N.; Wechsler, M.S.; McCloskey, E.A.; Yu, J.H.; Patricelli, G.L. Successfully mating male sage-grouse show greater laterality in courtship and aggressive interactions. Anim. Behav. 2016, 111, 261-267. [CrossRef]

26. Jennings, D.J. Right-sided bias in fallow deer terminating parallel walks: Evidence for lateralization during a lateral display. Anim. Behav. 2012, 83, 1427-1432. [CrossRef]

27. Camerlink, I.; Menneson, S.; Turner, S.P.; Farish, M.; Arnott, G. Lateralization influences contest behaviour in domestic pigs. Sci. Rep. 2018, 8, 12116. [CrossRef]

28. Found, R.; Clair, C.C. Ambidextrous ungulates have more flexible behaviour, bolder personalities and migrate less. R. Soc. Opensci. 2017, 4, 160958. [CrossRef]

29. Dharmaretnam, M.; Rogers, L.J. Hemispheric specialization and dual processing in strongly versus weakly lateralized chicks. Behav. Brain Res. 2005, 162, 62-70. [CrossRef]

30. Reddon, A.R.; Hurd, P.L. Individual differences in cerebral lateralization are associated with shy-bold variation in the convict cichlid. Anim. Behav. 2009, 77, 189-193. [CrossRef]

31. Byrnes, E.E.; Pouca, C.V.; Brown, C. Laterality strength is linked to stress reactivity in Port Jackson sharks (Heterodontus portusjacksoni). Behav. Brain Res. 2016, 305, 239-246. [CrossRef]

32. Whiteside, M.A.; Bess, M.M.; Frasnelli, E.; Beardsworth, C.E.; Langley, E.J.G.; van Horik, J.O.; Madden, J.R. Low survival of strongly footed pheasants may explain constraints on lateralization. Sci. Rep. 2018, 8, 13791. [CrossRef]

33. Hirnstein, M.; Leask, S.; Rose, J.; Hausmann, M. Disentangling the relationship between hemispheric asymmetry and cognitive performance. Brain Cogn. 2010, 73, 119-127. [CrossRef]

34. Boles, D.B.; Barth, J.M.; Merrill, E.C. Asymmetry and performance: Toward a neurodevelopmental theory. Brain Cogn. 2008, 66, 124-139. [CrossRef]

35. McManus, I.C. Right Hand, Left Hand: The Origins of Asymmetry in Brains, Bodies, Atoms, and Cultures; Weidenfeld \& Nicolson: London, UK, 2002. 
36. Rogers, L.J.; Workman, L. Light exposure during incubation affects competitive behaviour in domestic chicks. Appl. Anim. Behav. Sci. 1989, 23, 187-198. [CrossRef]

37. Ghirlanda, S.; Vallortigara, G. The evolution of brain lateralisation: A game-theoretical analysis of population structure. Proc. R. Soc. B 2004, 271, 853-857. [CrossRef]

38. Vallortigara, G. The evolutionary psychology of left and right: Costs and benefits of lateralization. Dev. Psychobiol. 2006, 48, 418-427. [CrossRef]

39. Lippolis, G.; Bisazza, A.; Rogers, L.J.; Vallortigara, G. Lateralization of predator avoidance responses in three species of toads. Laterality 2002, 7, 163-183. [CrossRef]

40. Foster, W.A.; Treherne, J.E. Evidence for the dilution effect in the selfish herd from fish predation of a marine insect. Nature 1981, 293, 508-510. [CrossRef]

41. Chivers, D.P.; McCormick, M.I.; Allan, B.J.; Mitchell, M.D.; Goncalves, E.J.; Bryshun, R.; Ferrari, M.C. At odds with the group: Changes in lateralization and escape performance reveal conformity and conflict in fish schools. Proc. R. Soc. Lond. B 2016, 283, 20161127. [CrossRef]

42. Ghirlanda, S.; Frasnelli, E.; Vallortigara, G. Intraspecific competition and coordination in the evolution of lateralization. Phil. Trans. R. Soc. Lond. B 2009, 364, 861-866. [CrossRef]

43. Loffing, F. Left- handedness and time pressure in elite interactive ball games. Biol. Lett. 2017, 13, 20170446. [CrossRef]

44. Faurie, C.; Raymond, M. Handedness, homicide and negative frequency-dependent selection. Proc. R. Soc. Lond. B 2005, 272, 25-28. [CrossRef]

45. Frasnelli, E.; Haase, A.; Rigosi, E.; Anfora, G.; Rogers, L.J.; Vallortigara, G. The bee as a model to investigate brain and behavioural asymmetries. Insects 2014, 5, 120-138. [CrossRef]

46. Niven, J.E.; Frasnelli, E. Insights into the evolution of lateralization from the insects. Progr. Brain Res. 2018, 238, 3-31.

47. Anfora, G.; Frasnelli, E.; Maccagnani, B.; Rogers, L.J.; Vallortigara, G. Behavioural and electrophysiological lateralization in a social (Apis mellifera) but not in a non-social (Osmia cornuta) species of bee. Behav. Brain Res. 2010, 206, 236-239. [CrossRef]

48. Frasnelli, E.; Vallortigara, G.; Rogers, L.J. Right-left antennal asymmetry of odour memory recall in three species of Australian stingless bees. Behav. Brain Res. 2011, 224, 121-127. [CrossRef]

49. Anfora, G.; Rigosi, E.; Frasnelli, E.; Trona, F.; Vallortigara, G. Lateralization in the invertebrate brain: Left-right asymmetry of olfaction in bumble bee, Bombus terrestris. PLoS ONE 2011, 6, e18903. [CrossRef]

50. Frasnelli, E.; Iakovlev, I.; Reznikova, Z. Asymmetry in antennal contacts during trophallaxis in ants. Behav. Brain Res. 2012, 232, 7-12. [CrossRef]

51. Rogers, L.J.; Rigosi, E.; Frasnelli, E.; Vallortigara, G. A right antenna for social behaviour in honeybees. Sci. Rep. 2013, 3, 2045. [CrossRef]

52. Rogers, L.J.; Frasnelli, E. Antennal Asymmetry in Social Behavior of the Australian Stingless Bee, Tetragonula carbonaria. J. Insect Behav. 2016, 29, 491-499. [CrossRef]

53. Karenina, K.; Giljov, A.; Ingram, J.; Rowntree, V.J.; Malashichev, Y. Lateralization of mother-infant interactions in a diverse range of mammal species. Nat. Ecol. Evol. 2017, 1, 0030. [CrossRef]

54. Rogers, L.J.; Frasnelli, E.; Versace, E. Lateralized antennal control of aggression and sex differences in red mason bees, Osmia bicornis. Sci. Rep. 2016, 6, 29411. [CrossRef]

55. Letzkus, P.; Boeddeker, N.; Wood, J.T.; Zhang, S.W.; Srinivasan, M.V. Lateralization of visual learning in the honeybee. Biol. Lett. 2008, 4, 16-19. [CrossRef]

56. Letzkus, P.; Ribi, W.A.; Wood, J.T.; Zhu, H.; Zhang, S.W.; Srinivasan, M.V. Lateralization of olfaction in the honeybee Apis mellifera. Curr. Biol. 2006, 16, 1471-1476. [CrossRef]

57. Rogers, L.J.; Vallortigara, G. From antenna to antenna: Lateral shift of olfactory memory recall by honeybees. PLoS ONE 2008, 3, e2340. [CrossRef]

58. Frasnelli, E.; Vallortigara, G.; Rogers, L.J. Response competition associated with right-left antennal asymmetries of new and old olfactory memory traces in honeybees. Behav. Brain Res. 2010, 209, 36-41. [CrossRef]

59. Ong, M.; Bulmer, M.; Groening, J.; Srinivasan, M.V. Obstacle traversal and route choice in flying honeybees: Evidence for individual handedness. PLoS ONE 2017, 12, e0184343. [CrossRef]

60. Hönicke, C.; Bliss, P.; Moritz, R.F. Effect of density on traffic and velocity on trunk trails of Formica pratensis. Sci. Nat. 2015, 102, 17. [CrossRef] 
61. Calcraft, P.R.T.; Bell, A.T.; Husbands, P.; Philippides, A.; Niven, J.E. The evolution of handedness: Why are ant colonies left-and right-handed? Biomath Commun. 2016, 3. [CrossRef]

62. Benelli, G.; Donati, E.; Romano, D.; Stefanini, C.; Messing, R.H.; Canale, A. Lateralisation of aggressive displays in a tephritid fly. Sci. Nat. 2015, 102, 1. [CrossRef]

63. Downes, J.C.; Birsoy, B.; Chipman, K.C.; Rothman, J.H. Handedness of a motor program in C. elegans is independent of left-right body asymmetry. PLoS ONE 2012, 7, e52138. [CrossRef]

64. Kurvers, R.H.; Krause, S.; Viblanc, P.E.; Herbert-Read, J.E.; Zaslansky, P.; Domenici, P.; Couillaud, P. The evolution of lateralization in group hunting sailfish. Curr. Biol. 2017, 27, 521-526. [CrossRef]

65. Frasnelli, E. Lateralization in Invertebrates. In Lateralized Brain Functions: Methods in Human and Non-Human Species; Neuromethods; Springer Protocols; Rogers, L.J., Vallortigara, G., Eds.; Humana Press: New York, NY, USA, 2017; Volume 122, pp. 153-208.

(c) 2018 by the authors. Licensee MDPI, Basel, Switzerland. This article is an open access article distributed under the terms and conditions of the Creative Commons Attribution (CC BY) license (http://creativecommons.org/licenses/by/4.0/). 